\title{
PROINOV CONTRACTIONS AND DISCONTINUITY AT FIXED POINT
}

\author{
RAVINDRA K. BISHT, R. P. PANT, AND VLADIMIR RAKOČEVIĆ
}

Received 14 March, 2017

\begin{abstract}
In this paper, we show that the contractive definition considered by Proinov [Fixed point theorems in metric spaces, Nonlinear Analysis 64 (2006) 546 - 557] is strong enough to generate a fixed point but does not force the mapping to be continuous at the fixed point. Thus we provide several answers to the open question posed by B.E. Rhoades in Contractive definitions and continuity, Contemporary Mathematics 72(1988), 233-245.
\end{abstract}

2010 Mathematics Subject Classification: 47H09; 54E50; 47H10; 54E40

Keywords: fixed point, $(\epsilon-\delta)$ contractions, power contraction, orbital continuity

\section{INTRODUCTION}

For a self-mapping $T$ of a metric space $(X, d)$ we put

$$
m(x, y)=\max \left\{d(x, y), d(x, T x), d(y, T y), \frac{d(x, T y)+d(y, T x)}{2}\right\} .
$$

The most general type of contractive condition is either a Ćirić [7] type contractive condition

$$
d(T x, T y) \leq \alpha \cdot m(x, y), x, y \in X, 0 \leq \alpha<1,
$$

or a $\varphi$ - contractive condition (see $[4,6,8,9]$ ) of the form

$$
d(T x, T y) \leq \varphi(m(x, y)), x, y \in X,
$$

where $\varphi: \mathbb{R}_{+} \rightarrow \mathbb{R}_{+}$satisfies different set of conditions (see [1,18,23,24]), or a MeirKeeler [15] type $(\epsilon, \delta)$ contractive condition,

given $\epsilon>0$ there exists a $\delta(\epsilon)>0$ such that for $x, y \in X$,

$$
\epsilon \leq m(x, y)<\epsilon+\delta \Longrightarrow d(T x, T y)<\epsilon .
$$

Let us recall that a fixed point of $T$ is said to be contractive [12] if all of the Picard iterates of $T$ converge to this fixed point. The set $O(x ; T)=\left\{T^{n} x: n=0,1,2, \ldots\right\}$ is called the orbit of the self-mapping $T$ at the point $x \in X$. Mapping $T$ is orbitally continuous at a point $z \in X$ if for any sequence $\left\{x_{n}\right\} \subset O(x ; T)$ (for some $\left.x \in X\right)$ $x_{n} \rightarrow z$ implies $T x_{n} \rightarrow T z$ as $n \rightarrow \infty$. Every continuous self-mapping of a metric 
space is orbitally continuous, but the converse need not be true (see Example 2.1 below). Mapping $T$ is asymptotically regular if $\lim _{n \rightarrow \infty} d\left(T^{n} x, T^{n+1} x\right)=0$ for each $x \in X[5]$.

Let $\Phi_{1}$ [21] denote the class of all functions $\varphi: \mathbb{R}_{+} \rightarrow \mathbb{R}_{+}$satisfying; for any $\epsilon>0$ there exists $\delta>\epsilon$ such that $\epsilon<t<\delta$ implies $\varphi(t) \leq \epsilon$. It is easy to see that $\varphi(t)<t$ for $t>0$. In 2006, Proinov [21] proved the following very interesting fixed point theorem which subsumes most of the fixed point theorems based on conditions discussed above.

Theorem 1. Let $(X, d)$ be a complete metric space. Let $T$ be a continuous and asymptotically regular self-mapping on $X$ such that:

(i) There exists $\varphi \in \Phi_{1}$ such that $d(T x, T y) \leq \varphi\left(D_{\gamma}(x, y)\right)$ for all $x, y \in X$, where $D_{\gamma}(x, y)=d(x, y)+\gamma[d(x, T x)+d(y, T y)]$, where $\gamma \geq 0$;

(ii) $d(T x, T y)<D_{\gamma}(x, y)$ for all $x, y \in X$ with $x \neq y$.

Then $T$ has a contractive fixed point. Moreover, in the case $\gamma=1$ and if $\varphi$ is continuous and satisfies $\varphi(t)<t$ for all $t>0$, then the continuity of $T$ can be dropped.

Let us point out (see Theorem 3.2 [21]), that the first part of Theorem 1 is equivalent to the following result.

Theorem 2. Let $(X, d)$ be a complete metric space. Let $T$ be a continuous and asymptotically regular self-mapping on $X$ such that:

(i) For any $\epsilon>0$ there exists $\delta>\epsilon$ such that $\epsilon<D_{\gamma}(x, y)<\delta$ implies $d(T x, T y) \leq \epsilon$

(ii) $d(T x, T y)<D_{\gamma}(x, y)$ for all $x, y \in X$ with $x \neq y$.

Then $T$ has a contractive fixed point.

The question whether there exists a contractive definition which is strong enough to generate a fixed point but which does not force the mapping to be continuous at the fixed point was investigated by Rhoades in [24] as an existing open problem. The question of the existence of contractive mappings which are discontinuous at their fixed points was settled in the affirmative by Pant [16]. In order to achieve his goal he employed a combination of an $(\epsilon-\delta)$ condition and a $\phi$-contractive condition to prove a fixed point in which the fixed point may be a point of discontinuity. Recently in [3] Bisht and Rakočević have studied generalized Meir-Keeler type contractions and discontinuity at fixed point.

In this paper we show that the contractive definition introduced by Proinov [21] need not be continuous at the fixed point. Thus we not only relax the continuity requirement in the results proved by Proinov but also provide more answers to the open question posed in [24]. 


\section{MAIN RESULTS}

Our first main result is the following.

Theorem 3. Let $(X, d)$ be a complete metric space. Let $T$ be an orbitally continuous and asymptotically regular self-mapping on $X$ satisfying ( $i$ ) and (ii) of Theorem 1. Then $T$ has a contractive fixed point.

Proof. Let $x_{0}$ be any point in $X$. Define a sequence $\left\{x_{n}\right\}$ in $X$ given by the rule $x_{n+1}=T^{n} x_{0}=T x_{n}$. From the proof of Theorem 4.1 of [21], we know that $\lim _{n} d\left(x_{n}, x_{n+1}\right)=0$ and that $\left\{x_{n}\right\}$ is a Cauchy sequence. Since $X$ is complete, there exists a point $z \in X$ such that $x_{n} \rightarrow z$ as $n \rightarrow \infty$. Also $T x_{n} \rightarrow z$. Orbital continuity of $T$ implies that $\lim _{n \rightarrow \infty} T x_{n}=T z$. This yields $T z=z$, that is, $z$ is a fixed point of $T$. Uniqueness of the fixed point follows from (ii).

As in Theorem 2, the first part of Theorem 3 is equivalent to the following result.

Theorem 4. Let $(X, d)$ be a complete metric space. Let $T$ be an orbitally continuous and asymptotically regular self-mapping on $X$ such that:

(i) For any $\epsilon>0$ there exists $\delta>\epsilon$ such that $\epsilon<D_{\gamma}(x, y)<\delta$ implies $d(T x, T y) \leq \epsilon$

(ii) $d(T x, T y)<D_{\gamma}(x, y)$ for all $x, y \in X$ with $x \neq y$.

Then $T$ has a contractive fixed point.

The following example [16] illustrates the above theorems.

Example 1. Let $X=[0,2]$ and $d$ be the usual metric on $X$. Define $T: X \rightarrow X$ by

$$
T(x)=\left\{\begin{array}{l}
1, \text { if } 0 \leq x \leq 1 \\
0, \text { if } 1<x \leq 2
\end{array}\right.
$$

Then $T$ satisfies all the conditions of above theorems and has a unique fixed point $x=1$. The mapping $T$ satisfies condition (i) of Theorems 3 and 4 with

$$
\begin{gathered}
\varphi(t)= \begin{cases}1 & \text { if } t>1 ; \\
\frac{t}{2}, & \text { if } t \leq 1,\end{cases} \\
\delta(\epsilon)= \begin{cases}1 & \text { for } \epsilon \geq 1 ; \\
1-\epsilon, & \text { for } \epsilon<1, \text { respectively. }\end{cases}
\end{gathered}
$$

It can also be easily seen that $\lim _{x \rightarrow 1} D_{\gamma}(x, 1) \neq 0$ and $T$ is discontinuous at the fixed point $x=1$. It can be verified that $T$ neither satisfy Theorem 1 nor Theorem 2. Therefore, Theorems 3 and 4 are effective generalizations of Proinov fixed point theorem [21].

In the next theorem, we shall use the continuity of $T^{2}$ and a special case of $D_{\gamma}(x, y)$, that is $D_{1}(x, y)$.

Theorem 5. Let $(X, d)$ be a complete metric space. Let $T$ be an asymptotically regular self-mapping on $X$ such that $T^{2}$ is continuous for any $x, y \in X$; 
(i) There exists $\varphi \in \Phi_{1}$ such that $d(T x, T y) \leq \varphi\left(D_{1}(x, y)\right)$ for all $x, y \in X$ and $\varphi(t)<t$ for all $t>0$

(ii) $d(T x, T y)<D_{1}(x, y)$ for all $x, y \in X$ with $x \neq y$.

Then $T$ has a contractive fixed point. say $z$, and $T^{n} x \rightarrow z$ for each $x \in X$.

Proof. Let $x_{0}$ be any point in $X$. Define a sequence $\left\{x_{n}\right\}$ in $X$ given by the rule $x_{n+1}=T^{n} x_{0}=T x_{n}$. Then from the above theorem (taking $\gamma=1$ ) we conclude that $\left\{x_{n}\right\}$ is a Cauchy sequence. Since $X$ is complete, there exists a point $z \in X$ such that $x_{n} \rightarrow z$ as $n \rightarrow \infty$. Also $T x_{n} \rightarrow z$ and $T^{2} x_{n} \rightarrow z$. By continuity of $T^{2}$, we have $T^{2} x_{n} \rightarrow T^{2} z$. This implies $T^{2} z=z$. Using (i) we get

$$
d\left(T z, T^{2} x_{n}\right) \leq \varphi\left(D_{1}\left(z, T x_{n}\right)=\varphi\left(d\left(z, T x_{n}\right)+d(z, T z)+d\left(x_{n}, T^{2} x_{n}\right)\right) .\right.
$$

Taking $n \rightarrow \infty$ and in view of $\varphi(t)<t$ we get $d(z, T z)=0$, i.e., $z$ is a fixed point of $T$. Uniqueness of the fixed point follows from (ii).

As in Theorem 2 the first part of Theorem 5 is equivalent to the following result.

Theorem 6. Let $(X, d)$ be a complete metric space. Let $T$ be an asymptotically regular self-mapping on $X$ such that $T^{2}$ is continuous for any $x, y \in X$;

(i) For any $\epsilon>0$ there exists $\delta>\epsilon$ such that $\epsilon<D_{1}(x, y)<\delta$ implies $d(T x, T y) \leq \epsilon$

(ii) $d(T x, T y)<D_{1}(x, y)$ for all $x, y \in X$ with $x \neq y$.

Then $T$ has a contractive fixed point. say $z$, and $T^{n} x \rightarrow z$ for each $x \in X$.

The following theorems verify that power contraction also allows the possibility of discontinuity at the fixed point.

Theorem 7. Let $(X, d)$ be a complete metric space. Let $T$ be an asymptotically regular self-mapping on $X$ such that:

(i) There exists $\varphi \in \Phi_{1}$ such that

$$
d\left(T^{n} x, T^{n} y\right) \leq \varphi\left(d(x, y)+d\left(x, T^{n} x\right)+d\left(y, T^{n} y\right)\right)
$$

(ii)

$$
\text { for all } x, y \in X \text { and } n \in \mathbb{N} \text {; }
$$

$$
\begin{aligned}
& \qquad \begin{array}{l}
d\left(T^{n} x, T^{n} y\right) \\
\text { for all } x, y \in X \text { with } x \neq y .
\end{array} \\
& \text { f } y)+d\left(x, T^{n} x\right)+d\left(y, T^{n} y\right)
\end{aligned}
$$

Then $T$ has a contractive fixed point. say $z$, and $T^{n} x \rightarrow z$ for each $x \in X$.

Proof. By Theorem $1, T^{n}$ has a unique fixed point $z \in X$; i.e., $T^{n}(z)=z$. Then $T(z)=T\left(T^{n}(z)\right)=T^{n}(T(z))$ and so $T(z)$ is a fixed point of $T^{n}$. Since the fixed point of $T^{n}$ is unique, $T z=z$. To prove the uniqueness, we assume that $y$ is another fixed point of $T$. Then $T y=y$ and so $T^{n}(y)=y$. Again by the uniqueness of the fixed point of $T^{n}$, we have $z=y$. Hence $z$ is a fixed point of $T$.

As in Theorem 2, the first part of Theorem 7 is equivalent to the following result. 
Theorem 8. Let $(X, d)$ be a complete metric space. Let $T$ be an asymptotically regular self-mapping on $X$ such that:

(i) For any $\epsilon>0$ there exists $\delta>\epsilon$ such that $\epsilon<d(x, y)+d\left(x, T^{n} x\right)+$ $d\left(y, T^{n} y\right)<\delta$ implies $d\left(T^{n} x, T^{n} y\right) \leq \epsilon$ and $n \in \mathbb{N}$;

(ii) $d\left(T^{n} x, T^{n} y\right)<d(x, y)+d\left(x, T^{n} x\right)+d\left(y, T^{n} y\right)$ for all $x, y \in X$ with $x \neq y$.

Then $T$ has a contractive fixed point. say $z$, and $T^{n} x \rightarrow z$ for each $x \in X$.

In view of

$$
\begin{aligned}
m(x, y) & =\max \{d(x, y), d(x, T x), d(y, T y),[d(x, T y)+d(y, T x)] / 2\} \\
& \leq d(x, y)+d(x, T x)+d(y, T y),
\end{aligned}
$$

we get the following corollaries:

Corollary 1. Let $(X, d)$ be a complete metric space. Let $T$ be an orbitally continuous self-mapping on $X$ such that:

(i) There exists $\varphi \in \Phi_{1}$ such that $d(T x, T y) \leq \varphi(m(x, y))$ for all $x, y \in X$;

(ii) $d(T x, T y)<m(x, y)$ for all $x, y \in X$ with $x \neq y$.

Then $T$ has a contractive fixed point. say $z$, and $T^{n} x \rightarrow z$ for each $x \in X$.

Corollary 2. Let $(X, d)$ be a complete metric space. Let $T$ be an orbitally continuous self-mapping on $X$ such that:

(i) For any $\epsilon>0$ there exists $\delta>\epsilon$ such that $\epsilon<m(x, y)<\delta$ implies $d(T x, T y) \leq \epsilon$

(ii) $d(T x, T y)<m(x, y)$ for all $x, y \in X$ with $x \neq y$.

Then $T$ has a contractive fixed point. say $z$, and $T^{n} x \rightarrow z$ for each $x \in X$.

Corollary 3. Let $(X, d)$ be a complete metric space. Let $T$ be a self-mapping on $X$ such that $T^{2}$ is continuous for any $x, y \in X$;

(i) There exists $\varphi \in \Phi_{1}$ such that $d(T x, T y) \leq \varphi(m(x, y))$ for all $x, y \in X$;

(ii) $d(T x, T y)<m(x, y)$ for all $x, y \in X$ with $x \neq y$.

Then $T$ has a contractive fixed point. say $z$, and $T^{n} x \rightarrow z$ for each $x \in X$.

Corollary 4. Let $(X, d)$ be a complete metric space. Let $T$ be a self-mapping on $X$ such that $T^{2}$ is continuous for any $x, y \in X$;

(i) For any $\epsilon>0$ there exists $\delta>\epsilon$ such that $\epsilon<m(x, y)<\delta$ implies $d(T x, T y) \leq \epsilon$

(ii) $d(T x, T y)<m(x, y)$ for all $x, y \in X$ with $x \neq y$.

Then $T$ has a contractive fixed point. say $z$, and $T^{n} x \rightarrow z$ for each $x \in X$.

Remark 1 . The above proved theorems unify and improve the results due to Bisht and Pant [2], Boyd and Wong [4], Ćirić [7], Jachymski [8], Kannan [10], Lim [13], Kuczma et al. [11], Matkowski [14], Pant [16, 17], Park and Bae [19], Park and Rhoades [20], Proinov [21] and Rao and Rao [22]. 


\section{ACKNOWLEDGMENT}

The authors are thankful to the learned referees for suggesting some improvements and thereby removing certain obscurities in the presentation.

\section{REFERENCES}

[1] M. Abtahi, Z. Kadelburg, and S. Radenović, “ Fixed points of Ćiric-Matkowski-type contraction in $v$-generalized metric spaces.” RACSAM, vol. 111, pp. 57-64, 2017, doi: 10.1007/s13398-0160275-5.

[2] R. K. Bisht and R. P. Pant, “ A remark on discontinuity at fixed point.” J. Math. Anal. Appl., vol. 445, pp. 1239-1241, 2017, doi: 10.1016/j.jmaa.2016.02.053.

[3] R. K. Bisht and V. Rakočević, "Generalized Meir-Keeler type contractions and discontinuity at fixed point.” Fixed Point Theory, vol. 19, no. 1, pp. 57-64, 2018, doi: 10.24193/fpt-ro.2018.1.06.

[4] D. W. Boyd and J. S. Wong, “ On nonlinear contractions.” Proc. Amer. Math. Soc., vol. 20, no. 2, pp. 458-464, 1969, doi: 10.1090/S0002-9939-1969-0239559-9.

[5] F. E. Browder and W. V. Petryshyn, " The solution by iteration of nonlinear functional equations in Banach spaces.” Bull. Am. Math. Soc., vol. 72, no. 3, pp. 571-575, 1966, doi: 10.1090/S00029904-1966-11544-6.

[6] K. J. Chung, “ On fixed point theorems of Meir and Keeler.” Math. Japon., vol. 23, pp. 381-383, 1978.

[7] L. B. Cirić, "Generalized contractions and fixed point theorems." Publ. Inst. Math., vol. 26, pp. 19-26, 1971.

[8] J. Jachymski, "Equivalent conditions and Meir-Keeler type theorems." J. Math. Anal. Appl., vol. 194, pp. 293-303, 1995, doi: 10.1006/jmaa.1995.1299.

[9] G. Jungck, K. Moon, S. Park, and B. Rhoades, " On generalizations of the Meir-Keeler type contraction maps : Corrections." J. Math. Anal. Appl., vol. 180, pp. 221-222, 1993, doi: 10.1006/jmaa.1993.1397.

[10] R. Kannan, “Some results on fixed points-II.” Amer. Math. Mon., vol. 76, no. 4, pp. 405-408, 1969, doi: 10.1080/00029890.1969.12000228.

[11] M. Kuczma, B. Choczewski, and R. Ger, Iterative Functional Equations," Encyclopedia of Mathematics and its Applications. UK: Cambridge Univ. Press, Cambridge, 1990. doi: 10.1017/CBO9781139086639.

[12] S. Leader, "Fixed points for operators on metric spaces with conditional uniform equivalence of orbits." J. Math. Anal. Appl., vol. 61, pp. 466-474, 1977, doi: 10.1016/0022-247X(77)90131-7.

[13] T. C. Lim, “ On Characterizations of Meir-Keeler contractive maps.” Nonlinear Anal., vol. 46, pp. 113-120, 2001, doi: 10.1016/S0362-546X(99)00448-4.

[14] J. Matkowski, “Integrable solutions of functional equations.” Diss. Math., vol. 127, pp. 1-68, 1975.

[15] A. Meir and E. Keeler, “ A theorem on contraction mappings.” J. Math. Anal. Appl., vol. 28, pp. 326-329, 1969, doi: 10.1016/0022-247X(69)90031-6.

[16] R. P. Pant, “ Discontinuity and fixed points.” J. Math. Anal. Appl., vol. 240, pp. 284-289, 1999, doi: 10.1006/jmaa.1999.6560.

[17] R. P. Pant, "Noncompatible mappings and common fixed points." Soochow J. Math., vol. 26, pp. 29-35, 2000.

[18] R. P. Pant, “ A comparison of contractive definitions.” J. Indian Math. Soc., vol. 72, pp. 241-249, 2005.

[19] S. Park and J. S. Bae, “ Extension of a fixed point theorem of Meir-Keeler.” Ark. Math., vol. 19, pp. 223-228, 1981, doi: 10.1007/BF02384479. 
[20] S. Park and B. Rhoades, “ Meir-Keeler type contractive conditions.” Math. Japon., vol. 26, pp. 13-20, 1981

[21] P. D. Proinov, “Fixed point theorems in metric spaces.” Nonlinear Anal., vol. 64, pp. 546-557, 2006, doi: 10.1016/j.na.2005.04.044.

[22] I. H. N. Rao and K. P. R. Rao, “Generalizations of fixed point theorems of Meir and Keeler type.” Indian J. Pure Appl. Math., vol. 16, pp. 1249-1262, 1985.

[23] B. E. Rhoades, "A comparison of various definitions of contractive mappings ." Transactions of Amer. Math. Soc., vol. 226, pp. 257-290, 1977, doi: 10.1090/S0002-9947-1977-0433430-4.

[24] B. E. Rhoades, "Contractive definitions and continuity." Contemporary Mathematics, vol. 72, pp. 235-245, 1988, doi: 10.1090/conm/072.

Authors' addresses

Ravindra K. Bisht

Department of Mathematics, National Defence Academy, Pune, India

E-mail address: ravindra.bisht@yahoo.com

R. P. Pant

Department of Mathematics, Kumaun University, Nainital, India

E-mail address: pant_rp@rediffmail.com

Vladimir Rakočević

University of Niš, Faculty of Sciences and Mathematics, Višegradska 33, 18000 Niš, Serbia

E-mail address: vrakocesbb.rs 